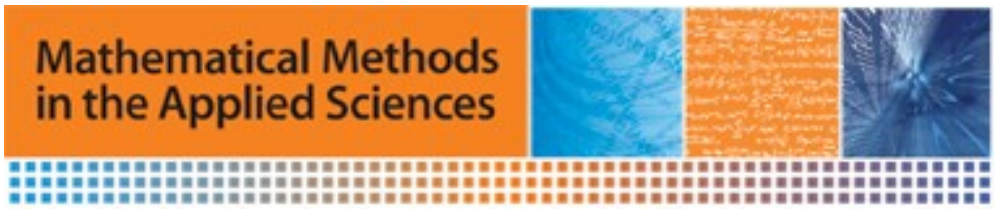

\title{
Uncertainty and sensitivity of the sexual behavior changes to the current Human Papillomavirus (HPV) vaccination campaign in Spain
}

\begin{tabular}{|r|l|}
\hline Journal: & Mathematical Methods in the Applied Sciences \\
\hline Manuscript ID & MMA-20-18286.R1 \\
\hline Wiley - Manuscript type: & Special Issue Paper \\
\hline Date Submitted by the & Author: \\
\hline Complete List of Authors: & $\begin{array}{l}\text { Acedo, Luis; University of Extremadura - Plasencia Campus, Mathematics } \\
\text { Cortés, Juan Carlos; Universidad Politécnica de Valencia, Instituto de } \\
\text { Matemática Multidisciplinar } \\
\text { Diez-Domingo, Javier; FISABIO-Public Health, Vaccine Research Unit } \\
\text { Sánchez-Alonso, Víctor; IMM, Instituto de Matemática Multidisciplinar } \\
\text { Tuells, Jose; University of Alicante, Department of Community Nursing, } \\
\text { Preventive Medicine and Public Health and History of Science } \\
\text { Villanueva, Rafael-Jacinto; Universitat Politecnica de Valencia, Instituto } \\
\text { Universitario de Matemática Multidisciplinar }\end{array}$ \\
\hline Keyword: & $\begin{array}{l}\text { Epidemiology, Computer model, Sensitivity analysis, Human } \\
\text { Papillomavirus (HPV) }\end{array}$ \\
\hline
\end{tabular}

\section{SCHOLARONE \\ Manuscripts}




\title{
Uncertainty and sensitivity of the sexual behavior changes to the current Human Papillomavirus $(\mathrm{HPV})$ vaccination campaign in Spain
}

\author{
Luis Acedo ${ }^{1}$, Juan-Carlos Cortés ${ }^{2}$, \\ Javier Díez-Domingo ${ }^{3}$, Víctor Sánchez-Alonso ${ }^{2}$, \\ José Tuells ${ }^{4}$, Rafael-J. Villanueva ${ }^{2}$ \\ ${ }^{1}$ Dept. Mathematics, Centro Universitario de Plasencia, \\ University of Extremadura, Plasencia, Spain \\ ${ }^{2}$ Instituto Universitario de Matemática Multidisciplinar, \\ $8 \mathrm{G}$ building, 2nd Floor, Camino de Vera s/n, \\ Universitat Politècnica de Valéncia, 46022 Valencia, Spain. \\ ${ }^{3}$ Vaccine Research Unit, Fundación para el Fomento de la \\ Investigación Sanitaria y Biomédica de la Comunitat \\ Valenciana, FISABIO-Public Health, Valencia, Spain \\ ${ }^{4}$ Department of Community Nursing, Preventive Medicine and \\ Public Health and History of Science, University of Alicante, \\ San Vicente del Raspeig, 03690 Alicante, Spain \\ email: acedo@unex.es, jccortes@imm.upv.es, \\ diez_jav@gva.es, vicsana3@doctor.upv.es, \\ tuells@ua.es, rjvillan@imm.upv.es.
}

\begin{abstract}
Taking into account the public health importance of the HPV control in the future, it is mandatory to assess the effect of the vaccination campaigns on the control of HPV spread and the associated diseases using reliable mathematical models. We propose a computational random network model with the aim of studying the transmission dynamics of HPV infections. This model reflects the herd immunity effect in the heterosexual network more accurately than the classical models. We perform a sensitivity analysis of the sexual behavior changes consisting of increasing the number of men who have sex with men (MSM), increasing the frequency of the intercourses and increasing the number of sexual partners.

${ }^{*}$ Corresponding author.
\end{abstract}


We find that large changes in the sexual behavior, in some extent, only have minor effects on the decline of the HPV infections in women and men in the current vaccination campaign in Spain (vaccination of young girls with a coverage of $70 \%$ ). Therefore, the current vaccination program in Spain is robust for the heterosexuals. However, we cannot say the same for MSM, where they do not benefit by the herd immunity effect of the vaccination of girls and, consequently, the circulation of the virus among them remains unchanged. A consequence of the present study is that the effect of other external factors that may affect the transmission dynamics of the HPV, for instance, the tourism or the immigration, do not influence the protection provided by the current Spanish vaccination program.

Keywords: HPV Transmission Dynamics, Computational Random Network Model, Sensitivity analysis, Public Health recommendations.

\section{Introduction}

Despite the importance in Public Health terms of sexually transmitted diseases (STD), we are confronted with the scarcity data to analyze sexual networks and, consequently, the lack of realistic representations. Therefore, it is not strange that the attempts to elucidate the structure of these networks are very limited both in scope and number of individuals considered $[1,2]$.

Also, the models usually proposed in literature to study HPV rely on systems of differential equations assuming the hypothesis of population homogeneous mixing [3], that is, any infectious individual can contact and infect any susceptible individual. Some of these models to study different aspects of the dynamics of HPV have been presented in $[4,5,6,7]$.

There is no doubt about the usefulness of classic models. However, in these models is very complex to distinguish among individuals, consider the transmission paths and consider possible relevant characteristics such as age, sex, previous illnesses or infections. Also, it is not clear that the assumption of the homogeneous mixing hypothesis be appropriate for STDs. Let us illustrate this issue with a simple example.

In Figure 1, we have a heterosexual network of sexual partners with 5 men $\left(M_{i}\right)$ and 5 women $\left(W_{i}\right)$ where women 2 and 3 are vaccinated, and consequently, protected against the infection. The lines, also called links or bonds, determine the sexual contacts, and consequently, the infection paths.

If homogeneous mixing is assumed, in any moment any man may be infected by an infected woman and vice versa. However, the structure of the network shows us that $M_{5}$ cannot infect any woman, because his sexual partner is vaccinated. Also, $M_{4}$ may infect $W_{4}$ and $W_{5}$ but not $W_{1}$. The longest infection path is $M_{2}-W_{4}-M_{4}-W_{5}$ and it gets blocked by the network structure and the vaccinated women $W_{2}$. Furthermore, if $W_{1}$ and $M_{1}$ are susceptible, they will remain protected by the herd immunity effect of the vaccinated $W_{2}$ and $W_{3}$. Thus, men and non-vaccinated women may be partially protected by vaccinated women and the sexual partners network structure.

This type of herd immunity due to the vaccination and the network structure is not considered in the classic models because of the assumption of the homogeneous mixing hypothesis. The presented example illustrates why these classic models are unable to explain situations as those happened in Australia $[8,9]$, meanwhile others, based on sexual partners network, can [10]. 


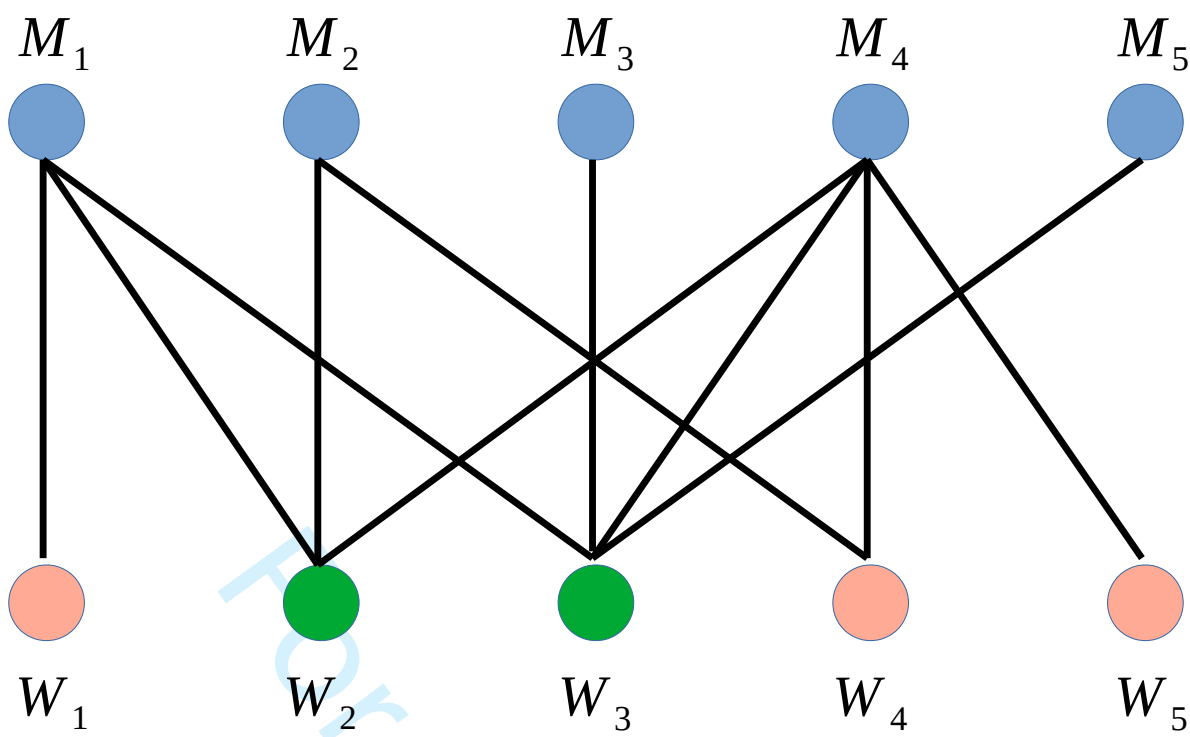

Figure 1: Heterosexual network of sexual partners. Women 2 and 3 are vaccinated and protect $M_{1}$ and $W_{1}$ by herd immunity effect from any infection coming from men 2,3,4 and 5, and women 4 and 5 because of the structure of the network.

In [10] we developed a HPV transmission dynamics model on a lifetime sexual partner's (LSP) network that uses individual contacts to monitor the evolution of the HPV infections, including features as the number of men who have sex with men (MSM), the number of LSP of each individual and the frequency of the intercourses; this allows us to provide a more detailed and realistic approach than the classic models. This computational random network model has a large number of nodes to study the HPV infections and the effects of the nine-valent vaccine on the short and long terms. As we mentioned, this model was able to reproduce the first results of the Australian vaccination campaign regarding the fast decline in the incidence of genital warts even after just two years of girls' vaccination $[8,9]$. This result was completely unexpected from the perspective of classical models and pointed towards an enhanced herd immunity effect in women and men as a result of the woman vaccination.

Although HPV immunization programs have been implemented in a lot of countries around the world, only a half of the 118 million women targeted have received at least a dose. This corresponds to the small amount of $1.7 \%$ of the total population. Also, the number of vaccinated women increases dramatically in developed regions [11].

In 2007, the Spanish National Health System passed the routine vaccination against HPV in an only cohort of girls aged $11-14$, and during the years $2007-2008$ the vaccination was implemented in all the autonomous communities. In 2015, the total global coverage in Spain was $79.2 \%$ [12]. In 2018 was decreased until $72.8 \%$ [13]. In this study we are going to consider a coverage for women of $70 \%$. Apart from the routine vaccination programs, there are specific recommendations in some Spanish autonomous communities in high-risk 
women's populations. Also, HPV vaccination in MSM is recommended up to age 26. Given the high burden of diseases related to genital warts, preferential use of quadrivalent or nine-valent vaccines is recommended.

Our main objective in this paper is to analyze the sensitivity of the current vaccination campaign in Spain to possible changes in the sexual behavior of the population. In particular, we are going to study the effect on the HPV infection of the increasing of the MSM population, the increasing the number of LSP and the frequency of intercourses, using a computational network model.

We must remark the difficulties that may appear when performing the present sensitivity study using classical models, because the number of LSP or the frequency of the intercourses are ususally embedded into the transmission parameter in such a way that to know marginal the effect of each one on the HPV infections may become troublesome.

The interest of this study emerges from the evidences of important changes in the sexual behaviour of Spanish women, that may induce changes in the HPV prevalence and the cervical cancer incidence in Spain [14]. Given that

- the important changes in the sexual behaviour of Spanish women [14],

- we have built the network with data from a statistical survey of 2003 [15] and we are in 2020 ,

- in this kind of surveys, men use to overestimate their number of sexual partners and women, in contrast, use to underestimate them,

it becomes relevant to perform a sensitivity analysis that supports the current vaccination campaign in Spain, taking into account the uncertainty of the above issues, and that guarantees that the model predictions in the medium and long-run, will not get significantly worse affected by changes in the sexual habits.

The interest of this study emerges from the fact that we have originally built the network with data from a statistical survey of 2003 [15]. Also, . Therefore, the obtained results may be biased and, then, may be easily extrapolated to the future as sexual habits might change.

In this paper, we perform that sensitivity analysis to yield a more substantiated prediction about the HPV in the Spanish present vaccination scenario, that is, vaccination of young girls with a coverage of $70 \%$, with the aim to see if the current vaccination strategy will return or not the expected benefits in case the sexual behavior changes. In other words, we want to check the robustness of the current vaccination programme in Spain.

\section{Methods}

\subsection{Computational network model building}

\subsubsection{Data}

A network consists of nodes (sites representing the individuals) and links or bonds between pairs of individuals, representing contacts (effective or not) for disease transmission. On a network, using computer programs, it is possible to simulate the evolution of the transmission of an infectious disease over the time. 
In order to build a realistic underlying demographic model, we consider the demographic data retrieved from [16], classified per age and sex, from 14 to 64 years old. A graphic representation of the demographic data can be seen in Figure 2.

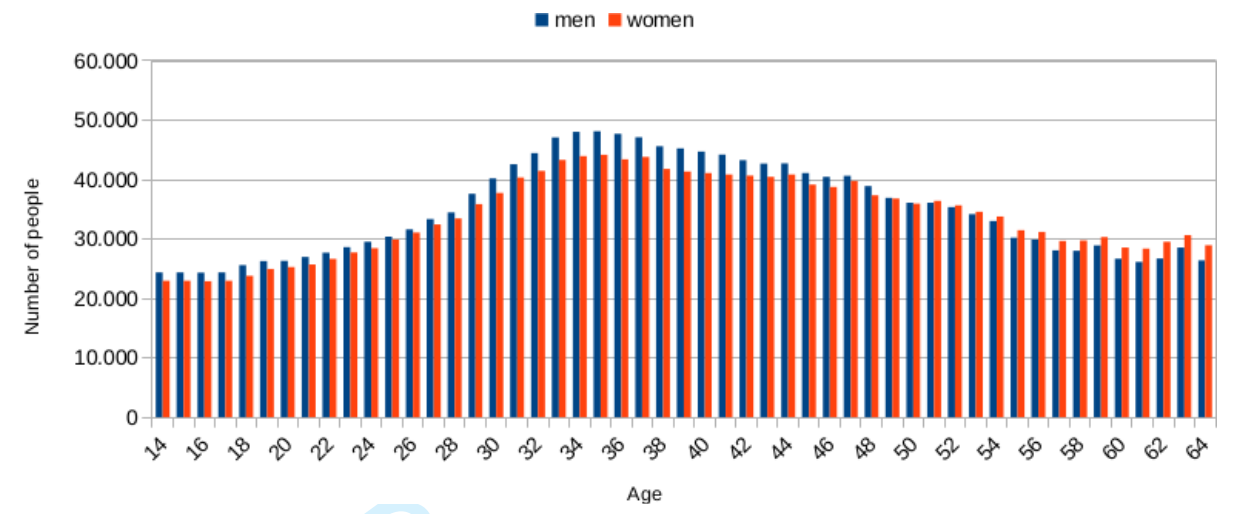

Figure 2: Number of men and women from 14 to 64 years old. $50.76 \%$ of people aged $14-64$ are men and $49.24 \%$ are women [16].

As we mentioned before, the main problem about building a realistic random network for sexually transmitted diseases is the lack of data. To our knowledge, there are neither recent general nor particular studies about the sexual behavior of the population. In the absence of enough experimental material to substantiate a particular type of network, we assumed that a large-scale network of sexual contacts could be similar to a random network in which individuals are characterized by a given number of contacts or bonds assigned randomly to other individuals. In reality, the assignment is not completely random but takes into account the similarity of individuals as explained below.

Concerning sexual habits of the population, the only information available to us is a survey conducted in Spain in 2003 with the objective of obtaining statistical information about the number of sexual partners until a certain age both for men and women [15]. The data is classified into age groups as displayed in Table 1.

\begin{tabular}{ccccccc} 
& \multicolumn{7}{c}{ MALES } \\
Age & 0 LSP & 1 LSP & 2 LSP & $3-4$ LSP & $5-9$ LSP & 10 or more LSP \\
\hline $14-29$ & 0.107 & 0.207 & 0.131 & 0.225 & 0.168 & 0.162 \\
$30-39$ & 0.027 & 0.225 & 0.128 & 0.210 & 0.170 & 0.240 \\
$40-65$ & 0.019 & 0.268 & 0.140 & 0.193 & 0.163 & 0.217 \\
\hline
\end{tabular}

\begin{tabular}{ccccccc} 
& \multicolumn{7}{c}{ FEMALES } \\
Age & 0 LSP & 1 LSP & 2 LSP & $3-4$ LSP & $5-9$ LSP & 10 or more LSP \\
\hline $14-29$ & 0.138 & 0.430 & 0.186 & 0.158 & 0.056 & 0.032 \\
$30-39$ & 0.029 & 0.501 & 0.168 & 0.177 & 0.077 & 0.048 \\
$40-65$ & 0.017 & 0.652 & 0.138 & 0.118 & 0.039 & 0.036 \\
\hline
\end{tabular}

Table 1: Proportion of males and females per number of lifetime sexual partners (LSP) per age group. Note that the sum of the rows is 1 . 
For each sex and each age group, Table 1 indicates the proportion of individuals with a given number of LSP from 0 to 10 or more. The LSP refers to the total number of sexual partners of an individual until the moment in which the survey was conducted.

The survey [15] says that $3.88 \%$ of MSM. MSM form a network themselves with high connectivity as shown data in Table 2 [17].

\begin{tabular}{c|cc|c} 
Age group & \# LSP & Age group & \# LSP \\
\hline $14-19$ & 18 & $40-49$ & 59 \\
$20-24$ & 25 & $50-59$ & 50 \\
$25-29$ & 33 & $60-85+$ & 56 \\
$30-39$ & 45 & Average & $\mathbf{3 9}$ \\
\hline
\end{tabular}

Table 2: Average number of LSP of MSM per age group and the global average $[17]$.

\subsubsection{Assigning sex, age and number of LSP to the nodes}

Let $N$ be the number of nodes of the network. For each node, we assign randomly sex (male or female) and age following the demographic structure given in Figure 2. Then, if the node is a

- male, with a probability 0.0388 the node is labeled as MSM and, taking into account its age, we assign its corresponding LSP following the data in Table 2. Otherwise, the node is considered as a heterosexual men and depending on its age a number of LSP is assigned following the data probabilities in Table 1;

- female and depending on its age a number of LSP is assigned following the probabilities in Table 1.

Once the nodes have been labeled, we are in a situation as the one drawn in Figure 3, where sexual partners still have not been assigned.

We must say that the assignment of LSP in Table 1 contains not only the uncertainty of the groups $3-4$ and $5-9$, but also the group 10 or more LSP. This uncertainty comes from clustering due to the lack of more precise sample information about LSP. For the former, we assign it choosing randomly one value of LSP in the interval. For the latter, it is necessary to introduce as a parameter the average number of LSP of the heterosexual men, $k$. When $k$ is set, it is possible to assign properly LSP to the nodes, considering the uncertainty, in such a way that the total number of men's partners be equal to the total number of women's partners. From [17], $k$ should be around 8 .

Until now, each node has been labeled as male, female or MSM, by age and LSP, but the sexual partners are not still paired.

\subsubsection{Pairing the nodes}

The goal now is to connect the nodes in such a way the number of LSP assigned to each node be satisfied. First, we build the heterosexual subnetwork using a pairing algorithm (Greedy Randomized Adaptive Search Procedure, GRASP) 


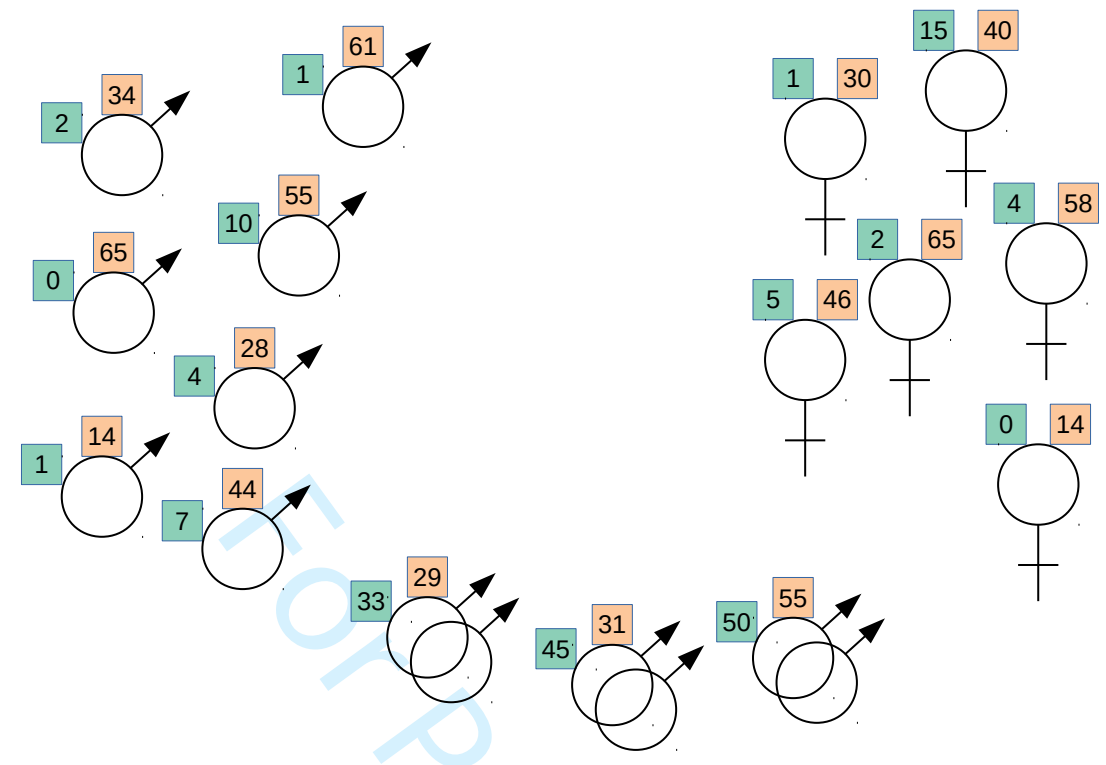

Figure 3: Example of nodes labeled. On the left are the heterosexual men. On the right, the women. The MSM are in the bottom. Each node is labeled by the number of LSP (green) and the age (orange). Note that the sexual partners are not still assigned.

[18] taking into account the principle of assortativity, that is, individuals with similar age and similar number of LSP are more likely to be connected. Second, MSM also constitute a subnetwork characterized by a larger number of average contacts [17] and also sporadic contacts with women [19] (bisexual men population). Unfortunately, to our knowledge, precise data about contacts bisexual men and women are unavailable. Therefore, we assume that each MSM may have a link with a woman with 5 or more LSP. This way, the subnetworks are not isolated because there is a bridge between the heterosexual and the MSM subnetworks where the infection may circulate. Thus, we build the MSM subnetwork using the GRASP algorithm and pairing the MSM nodes fulfilling their number of assigned LSP. Then, for each node MSM, we assign a link between it and a randomly chosen node women with 5 LSP or more.

An example of a small LSP network can be seen in Figure 4. More technical details for building the sexual contact network are described in previous publications $[10,20]$.

The built network is static. However, it is also important, in order to obtain simulations as realistic as possible, to take into account its evolution. To do so, we assume constant population (nobody dies, nobody is born and the old nodes are recycled as young 14-years-old nodes when they turn 65) and adapt the sexual behavior when the node grows and changes to a new age group. As the individuals grow up, we update the number of bonds in such a way that the statistics in Table 1 are preserved. 


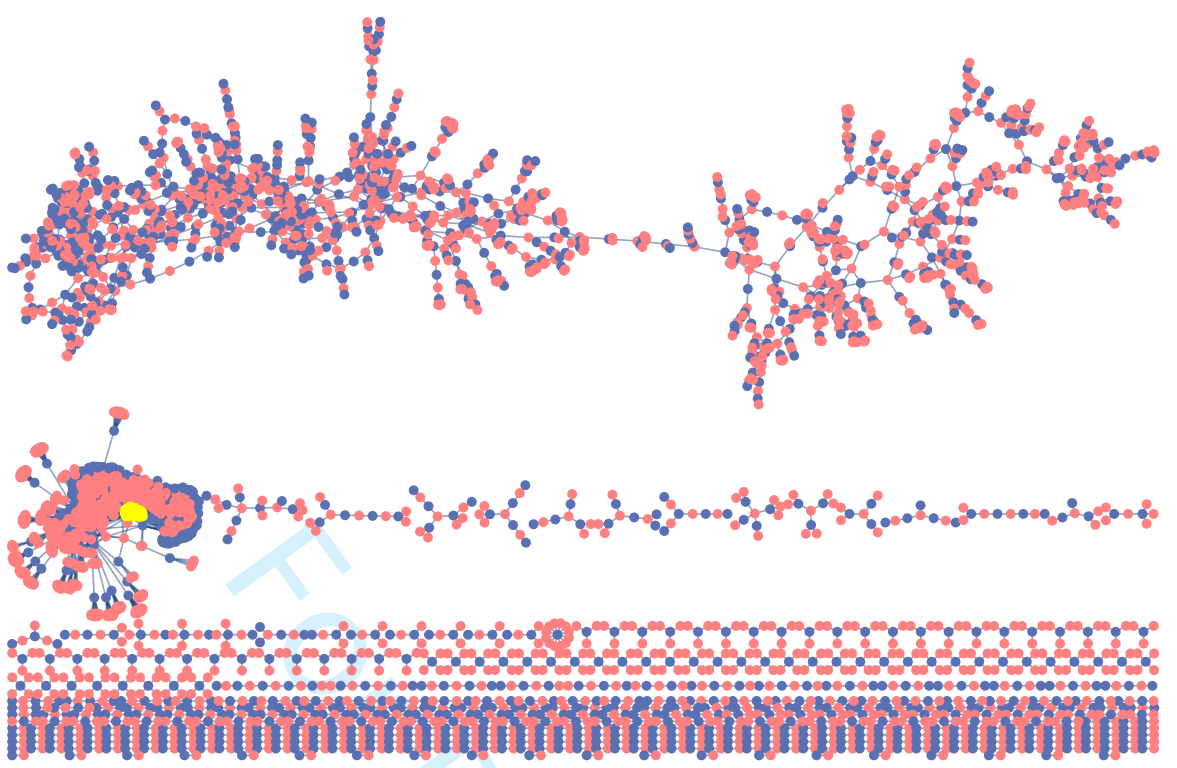

Figure 4: LSP network with 5000 nodes. Pink points are women, blue points heterosexual men and yellow points MSM. The lower part correspond to smallests subnetworks.

\subsubsection{HPV transmission dynamics}

Our model also takes into account infections by the most common genotypes in Spain classified into two categories:

- The HPV genotypes that causes cancer, also known as high risk (HR) or oncogenic; among these we consider for the model the genotypes 16/18/31/ $33 / 45 / 52 / 58$, because they are responsible of $90 \%$ of the HPV-related cancers [21] and the nine-valent vaccine protects against them.

- The HPV genotypes that causes genital warts, also known as low risk (LR); among these we consider for the model the genotypes 6/11, because they are responsible of $90 \%$ of the HPV-related genital warts [21] and the nine-valent vaccine protects against them.

The evolution of the spread of the HPV infections is simulated with a discrete version of the susceptible-infected-susceptible (SIS) model in which every node may be susceptible to the disease, infected by HR or LR genotypes or infected by both (co-infection).

A set of probabilities is also needed and calibrated to take into account the transitions among these states:

- Global probabilities that determine if the existence of a LSP implies intercourses in the current time step per age group 14 - 17, 18 - 29, 30 39 and 40 - 65. These parameters measure the global frequency of the intercourses per age group and may be different for each age group.

- The average recovery (clearing) times from a HR or LR infection. 
- Four parameters determining if the HR or LR infection is transmitted from a man or woman to his/her partner.

The simulation of the contagion is made through the transmission parameters, with certain probabilities. Then, in order to see if a contagion has been carried out by an intercourse (two connected nodes), we simulate this event by generating random numbers and checking if they are less than the corresponding thresholds given by the model transmission parameters. If it is, the susceptible node gets infected; otherwise, it remains susceptible. Therefore, randomness is included into the model in a natural way, in an attempt to perform as much as possible realistic simulations.

It should be noted that, in the computational network model, we have included explicitly parameters related to the number of LSP (network building), the frequency of the intercourses (HPV dynamics) and the percentage of MSM (network building). The variation of these parameters will allow us to perform the sensitivity analysis.

To calibrate the model, we use data retrieved from the CLEOPATRE study [22] in which the prevalence of different HPV genotypes in women was determined for a selected population in Spain. To the best of our knowledge, there are not more recent data prevalence. We use for men the same initial values (year 2007) as women because we do not have prevalence data for men in 2007. Data of 18-69 adult men have been recently reported for the years 2013-2014 in USA $[23,24]$. Our simulations returned similar values of prevalence for any HPV, HR and co-infection as those in $[23,24]$ for $2013-2014$. We cannot say the same for HPV LR where the difference is around $13 \%$.

This calibration has been performed probabilistically, returning $95 \%$ confidence intervals of the model parameter values and the model outputs. Details of the calibration can be found in [20] and the obtained results can be seen in Table 3. The obtained model parameter values are in agreement with the medical literature, for instance, [17] for the average LSP in men or $[4,25,26]$ for HPV clearing time.

We have used networks with a large number of nodes (100 000 for these problems) jointly with the calibrated model parameter values, to simulate the vaccination scenarios of interest with the nine-valent vaccine and the evolution of the HPV infections over the time.

\subsection{Scenarios to be simulated}

Let us consider our computational network model where we simulate the situation before the vaccination. We consider the year 0 when the vaccination program starts. The program consists of vaccinating young girls with a coverage of $70 \%$ in such a way that when they turn 14 years old and enter into the system, they are already vaccinated. Then, we perform the simulation in order to study the dynamics of the LR and HR over the next 100 years. Then, we calculate the decline of the prevalence of the LR and HR on women, heterosexual men and MSM over the time measured as

$$
D_{t}=100\left(1-\frac{P_{t}}{P}\right),
$$


Table 3: Mean and the $95 \%$ confidence interval (CI 95\%) of the model calibrated parameters [20]. Time is in years and probabilities are given as a fraction of unity. As we mentioned before, the "frequency" parameters represent global probabilities that determine if the existence of a LSP implies intercourses in the current time step per age group. The increasing of these probabilities imply an increase in the frequency of intercourses.

where $D_{t}$ is the decline of the LR (HR) in the time instant $t, P_{t}$ is the prevalence of the LR (HR) in the time instant $t$ and $P$ is the prevalence of the LR (HR) just before the vaccination program starts. The decline is measured as the percentual decrease of infected people with respect to the pre-vaccination scenario.

Here, we want to give an answer to the question: How is the decline of HPV affected if the percentage of MSM, the frequency of intercourses or the number of LSP increase significantly?

This sensitivity analysis is pertinent because the data used to build the network model and to perform the calibration have more than 15 years, and it is important to elucidate if significant changes in the sexual behavior will affect the current vaccination strategies substantially. This defines our current scenario corresponding to the sexual habits disclosed from Table 1 and a campaign of $\mathrm{HPV}$ vaccination targeting only on early female adolescents with a coverage around $70 \%$. Thus, we are going to simulate the changes in sexual behavior in three ways: increasing the percentages of MSM, increasing the frequency of the intercourses and increasing the number of LSP.

Thus, we propose the following scenarios to be simulated:

- Base Case. This is the current situation, with the LSP per age group as in Table 1, the frequency in the intercourses calibrated in [20], the percentage of MSM equal to $3.88 \%$, and the current program to vaccinate young girls with a coverage of $70 \%$.

- Case 1: MSM percentage increase. It is the Base Case increasing the percentage of MSM up to $10 \%$.

- Case 2: Increase of intercourses' frequency. It is the Base Case multiplying the model parameter values of intercourses' frequency per age groups by 2 . 
- Case 3: LSP's number increase. It is the Base Case where we are going to maintain the distribution percentages in Table 1 but changing the number of LSP. Thus, the proportion of people with only 1 LSP, in the simulation will have 2 ; people with 2 LSP will have 4 ; people with $3-4$ LSP will have 6-8; people with 5-9 LSP will have 9-13 and the people with 10 or more LSP will have 14 or more. Also, the number of LSP of MSM appearing in Table 2 will be increased by 4 .

\section{Results}

In this section we are going to compare the LR and HR declines of the prevalence in the Base Case with Cases 1, 2 and 3.

\subsection{Comparison Base Case and Case 1 (increase of the percentage of MSM until 10\%)}

In Case 1 there is an increase in the number of LSP of MSM around 160\%, compared to the Base Case. In Figures 5, 6 and 7 we can see graphically the comparison of the declines in the Base Case and the Case 1 for women, heterosexual men and men (here, men includes heterosexual men and MSM) and for MSM only, respectively.

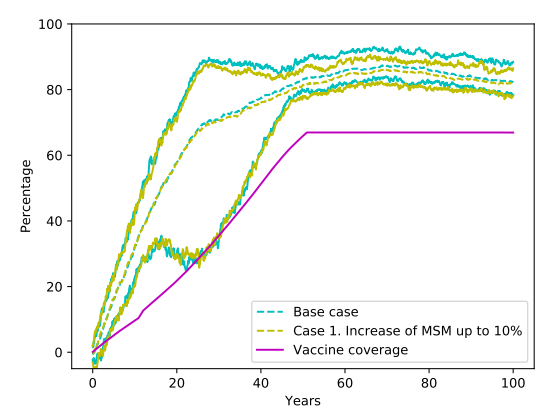

Decline of LR in women

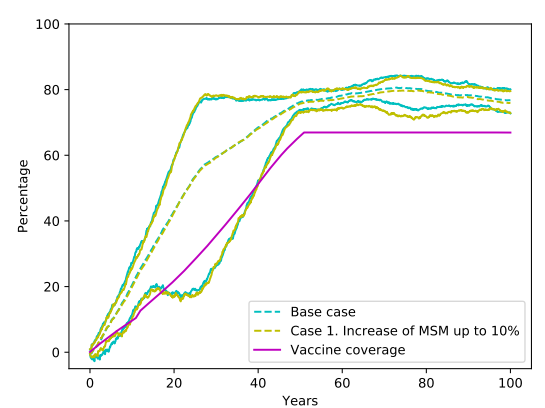

Decline of HR in women

Figure 5: Decline of LR and HR in women. Comparison between Base Case and Case 1 (increase of the percentage of MSM up to 10\%). Small differences can be seen in both scenarios (green and blue lines). Then, the increase of the percentage of MSM does not influence the decline in the HPV prevalence in women in the current vaccination program.

In the Figure 5 we can see that both scenarios show very similar declines over the time. To be precise, if we consider only the average decline in both cases along the 100 years of simulation, the maximum difference between the declines is $2.1 \%$ for LR and $1.2 \%$ for HR. Therefore, the increase of the MSM does not affect significantly the decline of HPV infections in women.

In the Figure 5, the magenta line shows the vaccine coverage. The difference between the lines of the decline and the line of the vaccine coverage measures the herd immunity effect of the vaccine to unvaccinated women. In average, this effect can be seen clearly from the very beginning. 
Figure 6 shows that the increase of the percentage of MSM only has influence on the MSM because the decline in heterosexual men hardly changes in both scenarios whereas there are significantly differences in the decline of men (including MSM). Considering the average declines in both scenarios along the whole simulation, the maximum difference of the declines between them are

- $2.3 \%$ for LR in heterosexual men,

- $1.1 \%$ for HR in heterosexual men,

- $18.2 \%$ for LR in men (MSM and heterosexual men),

- $7.0 \%$ for HR in men (MSM and heterosexual men).

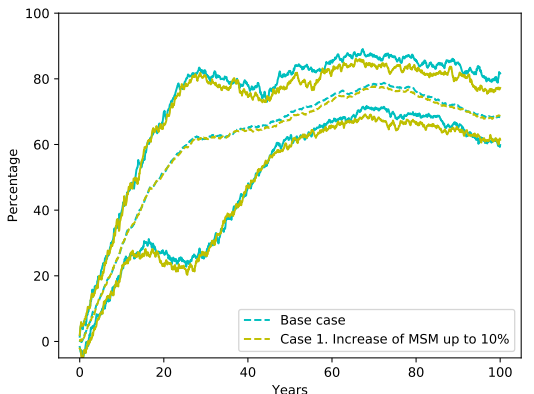

Decline of LR in heterosexual men

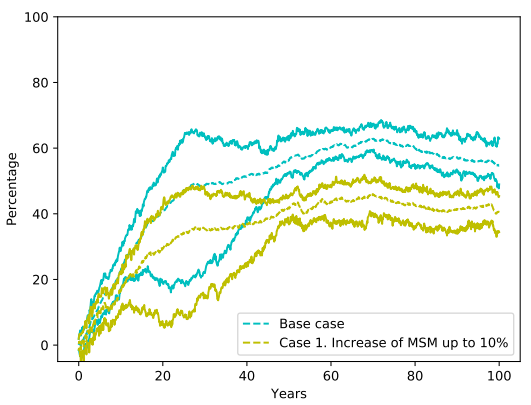

Decline of LR in all men

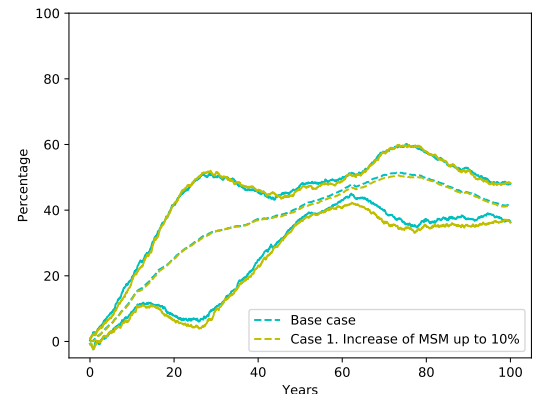

Decline of HR in heterosexual men

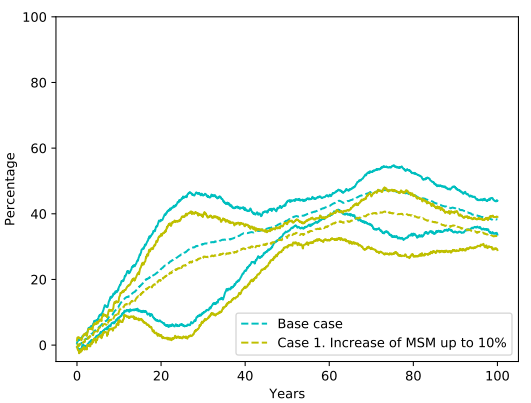

Decline of HR in all men

Figure 6: Decline of LR and HR in heterosexual men and all men (heterosexual and MSM). Comparison between Base Case and Case 1 (increase of the percentage of MSM up to 10\%). The increase in the decline in all the graphs are due to the herd immunity produced by the women's vaccination. The increase of MSM does not alter the decline in heterosexual men (upper figures) but it changes the decline in MSM, lower (green lines) when MSMs increase.

Note that the decline in men and in heterosexual men, is attributable to the herd immunity effect of the vaccination of women, because men are not vaccinated.

Figure 7 shows that the decline of HPV infections in MSM lies between -25\% and $20 \%$ with high uncertainty and without evidences of a sustained increasing 
trend in the decline. This means that, despite what happens with heterosexual men, the herd immunity provided by the vaccination of women does not have any impact on MSM. As the vaccination program is the same in all the simulations and due to the lack of herd immunity effect on MSM, it will not be necessary to show the behavior in the following comparisons because the result will be analogous to the one shown here in Figure 7.

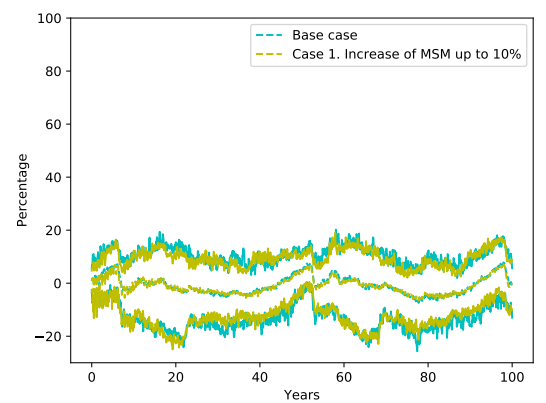

Decline of LR in MSM

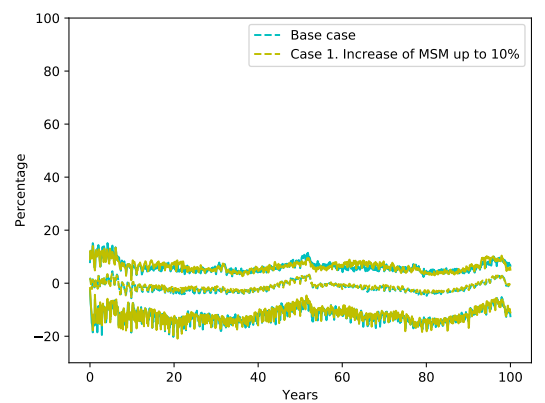

Decline of HR in MSM

Figure 7: Decline of LR and HR in MSM. Comparison between Base Case and Case 1 (increase of the percentage of MSM up to 10\%). Very small differences can be seen. No decline due to women's vaccination appears and this is very stable trend.

\subsection{Comparison Base Case and Case 2 (doubling the fre- quency of intercourses)}

In Figure 8 we can see graphically the comparison of the declines of Base Case and Case 2. The variations in the decline in women are small. There are some minor differences in men. In fact, if we consider the average declines in both scenarios along the whole simulation, the maximum difference of the declines between them are

- $6.0 \%$ for HR in men (MSM and heterosexual men),

- $6.7 \%$ for LR in men (MSM and heterosexual men),

- $4.0 \%$ for HR in women,

- $2.8 \%$ for LR in women.

The differences are more visible in the non-vaccinated population (men).

\subsection{Comparison Base Case and Case 3 (increasing the LSP)}

Grosso modo, in Case 3, we are increasing the number of heterosexual LSP in around $140 \%$ respect to the Base Case. In Figure 9 we can see graphically the comparison of the declines of HPV infections in Base Case and Case 3. Looking at the average declines in both scenarios along the 100 years simulation, the maximum difference between the declines are 


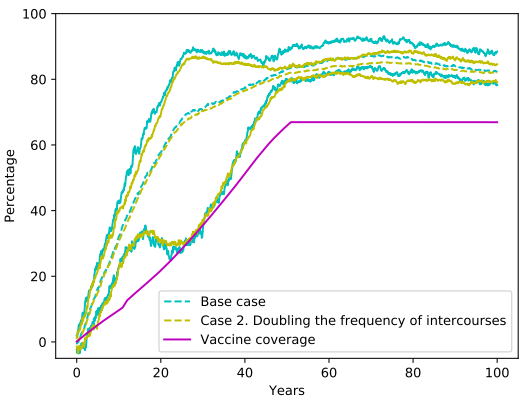

Decline of LR in women

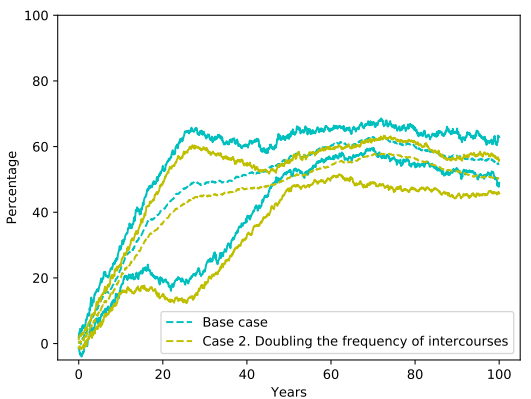

Decline of LR in all men

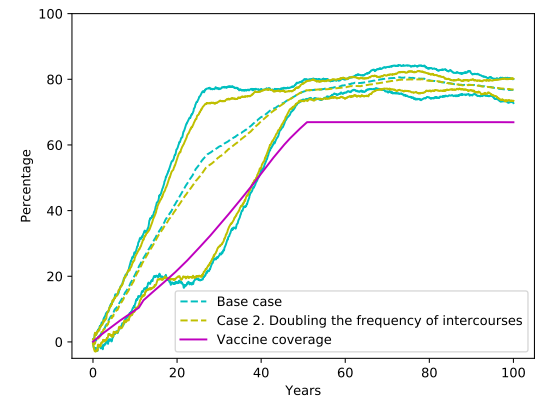

Decline of HR in women

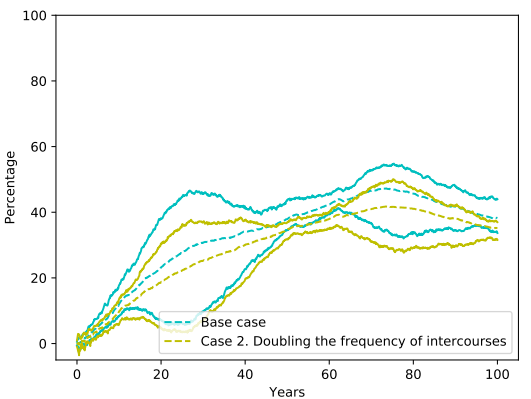

Decline of HR in all men

Figure 8: Decline of LR and HR in women and all men. Comparison between Base Case and Case 2. Small differences between declines appear.

- $6.7 \%$ for HR in men,

- $2.3 \%$ for LR in men,

- $5.3 \%$ for HR in women,

- $3.4 \%$ for LR in women.

The variations in the decline of $\mathrm{HR}$ in women and men are not large but greater than LR. In contrast with what happens in the previous comparisons, there is less variation in the decline of LR in women than in men. However, the levels of decline are much bigger in women (up to 84.5\%) than in men (up to $63.3 \%)$.

Figure 9 shows a small effect on the decline of the HPV infections despite the noticeable increase in the number of LSP. Therefore, although the sexual behavior may have changed in the last years and the number of LSP have increased, the herd immunity effect remains stable and it is not expected perceptible changes in the decline of the HPV infections.

\section{Discussion}

In Spain, currently, 11-14-years-old girls are being vaccinated against HPV in a routine vaccination program with a global coverage around $70 \%$ [13]. 


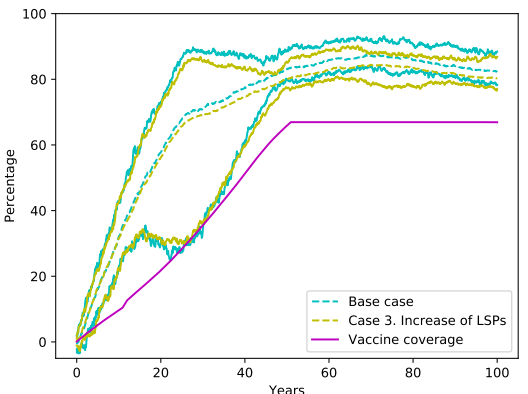

Decline of LR in women

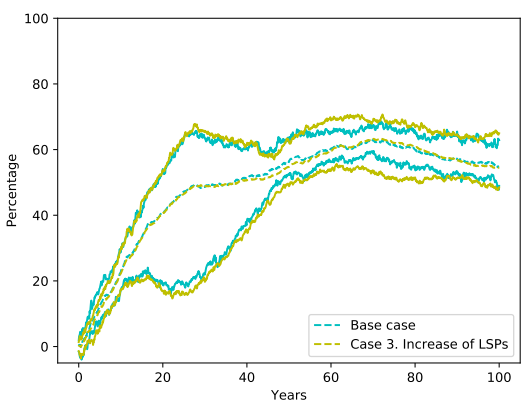

Decline of LR in men

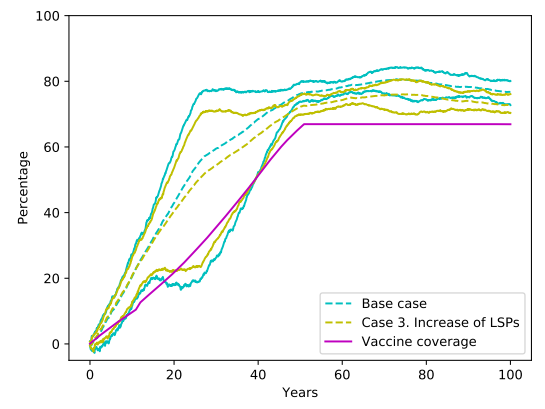

Decline of HR in women

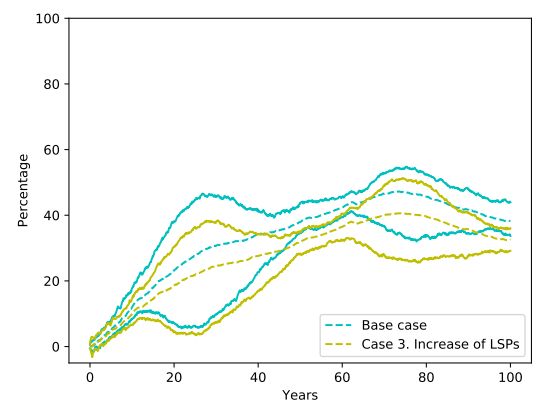

Decline of HR in men

Figure 9: Decline of LR and HR in women and all men. Comparison between Base Case and Case 3. There are only small changes in declines for oncogenic $\mathrm{HPV}$ in men.

Here, we have revisited a random network model for the transmission and control of HPV infections in Spain. We have previously shown that this model is more realistic than standard classic approaches and, in particular, it predicts the fast decline in genital warts in Australia only two years after the beginning of the vaccination campaign [10]. This happens because the computational network model is able to describe the herd immunity effect of the HPV vaccination programs more accurately than the classic models. The promising results obtained have motivated us to study different scenarios for vaccination in the middle and long terms. As it is generally known, these campaigns are targeting mainly the female population with doses administered at the late childhood or early adolescence.

Now, we propose the study of the sensitivity of the network model respect to the increase of the percentage of MSM, the increase of the frequency of the intercourses and the increase of the LSP. We must say that the proposed study would be difficult to be carried out with classical models because the parameters that govern the intercourses' frequency and the number of LSP are usually mixed with other parameters describing the transmission of the disease without a clear form to be separated with the aim to study the effect of their variation in the model output.

Our results show that a strong herd immunity effect would protect also the 
heterosexual male population as well as the unvaccinated women. We have also found that the MSM population is not exhibiting herd immunity effects in any measurable proportion and we can attribute this fact to their higher connectivity in the network and its non-bipartite character. These results have been found under the assumption that the average number of sexual partners for every age-group and sex remain the same given in the Sexual Habits Health Survey of 2003 [15]. Hence, it is important to consider new scenarios where the sexual habits change and check if these changes influence in some way on the expected vaccine protection.

For these reasons, in this paper we have focussed our study on the sensitivity of the vaccination campaigns' effectiveness to changes in the sexual habits. We have simulated these changes in three scenarios: increase of the MSM, increase in the intercourses' frequency and increase in the LSP. Our results show that the herd immunity effect is very robust against sexual behavior changes, given that the percentage of MSM, the intercourses' frequency and the number of LSP do not increase many times over their current estimated average values. This can also be explained by the fact that the heterosexual subnetwork is bipartite (as shown in Figure 1) and the vaccination of one sex places very stringent constraints on the propagation of the disease. In other nonsexual transmitted infectious diseases (respiratory or other) the propagation can occur among individuals of any age or sex and the herd immunity effect is not so evident. For instance, in Figure 2 of paper [27], the authors study the average number of contacts for the transmission dynamics of the Respiratory Syncytial Virus. They found that the average number of contacts should take values greater than 25 to explain the dynamics of the disease and the lack of the herd immunity effect. Therefore, looking at the average number of LSP in MSM given in Table 2 (39), we conjecture that it is necessary a very much strong increase of LSP in the heterosexual network than the simulated in this paper to observe whether the herd immunity effect, provided by the young girls vaccination, disappears.

As we see in the Figure 7, the MSM are completely unprotected when women are vaccinated. In some countries the incidence of anal cancer in men and cervical cancer in women are similar and therefore, some authors suggest targeting vaccination on MSM [28] to face the problem. Nevertheless, other authors claim that a program for MSM will not be able to target MSM before their sexual debut, or in fact even shortly after sexual debut because most MSM only disclose their sexual preference to health care providers in their early 20's [29]. Qendri et al. [30] have also studied the benefits of sex-neutral vaccination using a Bayesian framework and they have found that the extra protection gained by women (apart from the direct protection of MSM) could provide a reason to vaccinate also boys.

The present sensitivity study determines that the network model returns similar results when sexual behavior changes are simulated. As a consequence, we can extrapolate this result to elucidate that the effect of other external factors that may affect the transmission dynamics of the HPV, such as the tourism or the immigration, will not affect the expected results of the current Spanish vaccination program. 


\section{Acknowledgements}

- This work has been supported by the Spanish Ministerio de Economía, Industria y Competitividad (MINECO), the Agencia Estatal de Investigación (AEI) and Fondo Europeo de Desarrollo Regional (FEDER UE) grant MTM2017-89664-P.

- This paper has been supported by the European Union through the Operational Program of the [European Regional Development Fund (ERDF) / European Social Fund (ESF)] of the Valencian Community 2014-2020. Files: GJIDI/2018/A/010 and GJIDI/2018/A/009.
Abbreviations
STD Sexually transmitted disease
HPV Human papillomavirus
LSP Lifetime sexual partner
MSM Men having sex with men
HR High risk HPV
LR Low risk HPV
CI $95 \% \quad 95 \%$ confidence interval

The following abbreviations are used in this paper:

\section{References}

[1] P. S. Bearman, J. Moody, K. Stovel, Chains of affection: The structure of adolescent romantic and sexual networks, American Journal of Sociology 110 (1) (2004) 44-91. doi:10.1086/386272.

URL https://doi.org/10.1086/386272

[2] S. Helleringer, H.-P. Kohler, Sexual network structure and the spread of HIV in Africa: evidence from Likoma Island, Malawi, AIDS 21 (17) (2007) 2323-2332. doi:10.1097/qad.0b013e328285df98. URL https://doi.org/10.1097/qad.0b013e328285df98

[3] F. Brauer, C. Castillo-Chavez, Z. Feng, Mathematical Models in Epidemiology, Springer New York, 2019. doi:10.1007/978-1-4939-9828-9. URL https://doi.org/10.1007/978-1-4939-9828-9

[4] E. H. Elbasha, E. J. Dasbach, R. P. Insinga, Model for assessing human papillomavirus vaccination strategies, Emerging Infectious Diseases 13 (1) (2007) 28-41. doi:10.3201/eid1301.060438. URL https://doi.org/10.3201/eid1301.060438

[5] I. Baussano, K. M. Elfström, F. Lazzarato, A. Gillio-Tos, L. D. Marco, F. Carozzi, A. D. Mistro, J. Dillner, S. Franceschi, G. Ronco, Typespecific human papillomavirus biological features: Validated model-based estimates, PLoS ONE 8 (11) (2013) e81171. doi:10.1371/journal.pone. 0081171.

URL https://doi.org/10.1371/journal.pone.0081171 
[6] K. T. Simms, J. Steinberg, M. Caruana, M. A. Smith, J.-B. Lew, I. Soerjomataram, P. E. Castle, F. Bray, K. Canfell, Impact of scaled up human papillomavirus vaccination and cervical screening and the potential for global elimination of cervical cancer in 181 countries, 2020-99: a modelling study, The Lancet Oncology 20 (3) (2019) 394-407. doi:10.1016/s14702045 (18) 30836-2.

URL https://doi.org/10.1016/s1470-2045(18)30836-2

[7] M. Brisson, É. Bénard, M. Drolet, J. A. Bogaards, I. Baussano, S. Vänskä, M. Jit, M.-C. Boily, M. A. Smith, J. Berkhof, K. Canfell, H. W. Chesson, E. A. Burger, Y. H. Choi, B. F. D. Blasio, S. J. D. Vlas, G. Guzzetta, J. A. C. Hontelez, J. Horn, M. R. Jepsen, J. J. Kim, F. Lazzarato, S. M. Matthijsse, R. Mikolajczyk, A. Pavelyev, M. Pillsbury, L. A. Shafer, S. P. Tully, H. C. Turner, C. Usher, C. Walsh, Population-level impact, herd immunity, and elimination after human papillomavirus vaccination: a systematic review and meta-analysis of predictions from transmissiondynamic models, The Lancet Public Health 1 (1) (2016) e8-e17. doi: $10.1016 / \mathrm{s} 2468-2667(16) 30001-9$. URL https : //doi .org/10.1016/s2468-2667(16)30001-9

[8] C. K. Fairley, J. S. Hocking, L. C. Gurrin, M. Y. Chen, B. Donovan, C. S. Bradshaw, Rapid decline in presentations of genital warts after the implementation of a national quadrivalent human papillomavirus vaccination programme for young women, Sexually Transmitted Infections 85 (7) (2009) 499-502. doi:10.1136/sti.2009.037788. URL https://doi.org/10.1136/sti.2009.037788

[9] H. Ali, B. Donovan, H. Wand, T. R. H. Read, D. G. Regan, A. E. Grulich, C. K. Fairley, R. J. Guy, Genital warts in young Australians five years into national human papillomavirus vaccination programme: national surveillance data, BMJ 346 (apr18 1) (2013) f2032-f2032. doi: $10.1136 / \mathrm{bmj}$. f2032.

URL https://doi.org/10.1136/bmj.f2032

[10] J. Díez-Domingo, V. Sánchez-Alonso, R.-J. Villanueva, L. Acedo, J.-A. Moraño, J. Villanueva-Oller, Random network models to predict the longterm impact of HPV vaccination on genital warts, Viruses 9 (10) (2017) 300. doi:10.3390/v9100300.

URL https://doi.org/10.3390/v9100300

[11] L. Bruni, M. Diaz, L. Barrionuevo-Rosas, R. Herrero, F. Bray, F. X. Bosch, S. de Sanjosé, X. Castellsagué, Global estimates of human papillomavirus vaccination coverage by region and income level: a pooled analysis, The Lancet Global Health 4 (7) (2016) e453-e463. doi:10.1016/s2214$109 x$ (16) 30099-7.

URL https ://doi .org/10.1016/s2214-109x(16)30099-7

[12] L. Alemany, J. Bayas, N. Borruel, M. Campins, X. Castellsagué, A. Curran, C. D. de Heredia, X. Martínez, F. Moraga-Llop, A. Torné, AEPCC-Guía: Vacunación selectiva frente al virus del papiloma humano en poblaciones de riesgo elevado (Selective vaccination against the $\mathrm{HPV}$ in high risk populations), Publicaciones AEPCC, 2016. 
URL http://www.aepcc.org/wp-content/uploads/2016/12/AEPCC_ revista07_VACUNACION-SELECTIVA.pdf

[13] S. General de Promoción de la Salud y Vigilancia en Salud Pública, Coberturas de vacunación de la primera y segunda dosis de VPH en mujeres. comunidades autónomas. año 2018. (Vaccination coverages for the first and second HPV dose in women. autonomous Communities. year 2018, [Online; accessed 26 October 2019] (2018).

URL https://www.mscbs.gob.es/profesionales/saludPublica/ prevPromocion/vacunaciones/docs/CoberturasVacunacion/Tabla11. pdf

[14] S. de Sanjose, X. Cortés, C. Méndez, L. Puig-Tintore, A. Torné, E. Roura, F. Bosch, X. Castellsague, Age at sexual initiation and number of sexual partners in the female spanish population, European Journal of Obstetrics \& Gynecology and Reproductive Biology 140 (2) (2008) 234-240. doi: 10.1016/j. ejogrb.2008.04.005.

URL https://doi.org/10.1016/j.ejogrb.2008.04.005

[15] INE, Encuesta de Salud y Hábitos Sexuales. (Health and Sexual Habits Survey). Instituto Nacional de Estadística, [Online; accessed 6 March 2017] (2003).

URL http://www.ine.es/dyngs/INEbase/es/operacion.htm? c=Estadistica_C\&cid $=1254736176785 \&$ menu=resultados\&idp= $1254735573175 \mathrm{~g}$

[16] IVE, Portal estadistico de la Generalitat Valenciana (Statistical portal of the government of the Community of Valencia, Spain). Valencian Institute of Statistics, [Online; accessed 6 March 2017] (2013).

URL http://www.pegv.gva.es/va/padro-municipal-continuexplotacio-estadistica.-resultats-per-a-la-comunitatvalenciana

[17] Estudio de conducta sexual entre homosexuales (Study of sexual behavior among homosexuals), Technical Report, Durex (2002).

URL http://www.sidastudi.org/es/registro/ 2c9391e41fb402cc011fb442355a4176

[18] T. A. Feo, M. G. Resende, Greedy randomized adaptive search procedures, Journal of Global Optimization 6 (2) (1995) 109-133.

URL https://link.springer.com/article/10.1007/BF01096763

[19] M. Díaz, personal communication, Catalan Institute of Oncology (2016).

[20] R.-J. Villanueva, J. I. Hidalgo, C. Cervigón, J. Villanueva-Oller, J.-C. Cortés, Calibration of an agent-based simulation model to the data of women infected by human papillomavirus with uncertainty, Applied Soft Computing 80 (2019) 546-556. doi:10.1016/j.asoc.2019.04.015. URL https://doi.org/10.1016/j.asoc.2019.04.015

[21] S. Hartwig, J.-J. Baldauf, G. Dominiak-Felden, F. Simondon, L. Alemany, S. de Sanjosé, X. Castellsagué, Estimation of the epidemiological burden of HPV-related anogenital cancers, precancerous lesions, and genital warts 
in women and men in europe: Potential additional benefit of a nine-valent second generation HPV vaccine compared to first generation HPV vaccines, Papillomavirus Research 1 (2015) 90-100. doi:10.1016/j.pvr.2015.06. 003.

URL https://doi.org/10.1016/j.pvr.2015.06.003

[22] X. Castellsagué, T. Iftner, E. Roura, J. A. Vidart, S. K. Kjaer, F. X. Bosch, N. Muñoz, S. Palacios, M. S. M. Rodriguez, L. Serradell, L. Torcel-Pagnon, J. C. and, Prevalence and genotype distribution of human papillomavirus infection of the cervix in spain: The CLEOPATRE study, Journal of Medical Virology 84 (6) (2012) 947-956. doi:10.1002/jmv. 23282.

URL https://doi.org/10.1002/jmv. 23282

[23] J. J. Han, T. H. Beltran, J. W. Song, J. Klaric, Y. S. Choi, Prevalence of genital human papillomavirus infection and human papillomavirus vaccination rates among US adult men, JAMA Oncology 3 (6) (2017) 810. doi:10.1001/jamaoncol.2016.6192.

URL https://doi.org/10.1001/jamaoncol.2016.6192

[24] Centers for Disease Control and Prevention (CDC), Prevalence of HPV in adults aged 18-69: United states, 2011-2014 (2017).

URL https://www.cdc.gov/nchs/products/databriefs/db280.htm

[25] A. R. Giuliano, J.-H. Lee, W. Fulp, L. L. Villa, E. Lazcano, M. R. Papenfuss, M. Abrahamsen, J. Salmeron, G. M. Anic, D. E. Rollison, D. Smith, Incidence and clearance of genital human papillomavirus infection in men (HIM): a cohort study, The Lancet 377 (9769) (2011) 932-940. doi:10.1016/s0140-6736(10)62342-2.

URL https://doi.org/10.1016/s0140-6736(10)62342-2

[26] A. G. Nyitray, M. Chang, L. L. Villa, R. J. C. da Silva, M. L. Baggio, M. Abrahamsen, M. Papenfuss, M. Quiterio, J. Salmerón, E. LazcanoPonce, A. R. Giuliano, The natural history of genital human papillomavirus among HIV-negative men having sex with men and men having sex with women, Journal of Infectious Diseases 212 (2) (2015) 202-212. doi:10. 1093/infdis/jiv061.

URL https://doi.org/10.1093/infdis/jiv061

[27] L. Acedo, J.-A. Moraño, R.-J. Villanueva, J. Villanueva-Oller, J. DíezDomingo, Using random networks to study the dynamics of respiratory syncytial virus (RSV) in the spanish region of valencia, Mathematical and Computer Modelling 54 (7-8) (2011) 1650-1654. doi:10.1016/j.mcm. 2010.11 .068 .

URL https://doi.org/10.1016/j.mcm.2010.11.068

[28] J. A. Bogaards, S. H. Mooij, M. Xiridou, M. F. S. van der Loeff, Potential effectiveness of prophylactic HPV immunization for men who have sex with men in the netherlands: A multi-model approach, PLOS Medicine 16 (3) (2019) e1002756. doi:10.1371/journal.pmed.1002756.

URL https://doi.org/10.1371/journal.pmed.1002756 
[29] L. Zhang, D. G. Regan, J. J. Ong, M. Gambhir, E. P. Chow, H. Zou, M. Law, J. Hocking, C. K. Fairley, Targeted human papillomavirus vaccination for young men who have sex with men in Australia yields significant population benefits and is cost-effective, Vaccine 35 (37) (2017) 4923-4929. doi:10.1016/j.vaccine. 2017.07 .078 .

URL https://doi.org/10.1016/j.vaccine.2017.07.078

[30] V. Qendri, J. A. Bogaards, J. Berkhof, Who will benefit from expanding HPV vaccination programs to boys?, JNCI Cancer Spectrum 2 (4). doi: 10.1093/jncics/pky076.

URL https://doi.org/10.1093/jncics/pky076 